\title{
Seleção de espécies tolerantes para a fitorremediação de solo contaminado com imazapic
}

\author{
Selection of tolerant species for contaminated soil phytemedication using imazapic \\ Simonny Montthiel Araújo Vasconcelo, Adriano Jakelaitis*, Mailon Lucas Meurer Costa, Romário \\ Rodrigues Cunha de Oliveira, Vanessa Silva Santos
}

Instituto Federal Goiano, Rio Verde, GO, Brasil. *Autor para correspondência: adriano.jakelaitis@ifgoiano.edu.br

Submissão:04/05/2018 / Aceite: 06/03/2020

\begin{abstract}
RESUMO
O imazapic por apresentar longo efeito residual no solo pode ocasionar intoxicação visual em culturas sensíveis semeadas em rotação, além de representar riscos ecotoxicológicos em ambientes aquáticos. Diante disso, a fitorremediação é uma proposta inovadora como método alternativo para a remoção de poluentes orgânicos, sendo o sucesso da técnica dependente da seleção criteriosa de plantas com características favoráveis. O objetivo do estudo foi de identificar espécies de plantas tolerantes ao imazapic para potencial uso em programas de fitorremediação. Foram conduzidos oito experimentos em casa de vegetação climatizada com as espécies, Panicum maximum, Crotalaria juncea, Stylosanthes spp., Cajanus cajan, Dolichos lablab, Pennisetum glaucum, Mucuna aterrima, Raphanus sativus e cinco doses de imazapic $\left(0 ; 58,33 ; 87,5 ; 175\right.$ e $\left.350 \mathrm{~g} \mathrm{ha}^{-1}\right)$. Os tratamentos foram delineados em blocos ao acaso com quatro repetições. Aos 30 e 60 dias após a emergência das plantas foram avaliados os sintomas de intoxicação visual, altura e a massa seca das plantas, determinada ao término do experimento. As espécies $M$. aterrima, $C$. cajan e $D$. lablab não apresentaram sintomas visuais de fitointoxicação. $M$. aterrima foi a que promoveu maior produção de massa seca quando cultivada como testemunha e a exposição ao imazapic pouco afetou essa característica. Nas espécies tolerantes, a redução da altura e massa seca exigiu doses maiores, embora os resultados não tenham sido significativos para $D$. lablab e $C$. cajan. Entre essas espécies, $M$. aterrima se destacou pela alta produção de biomassa, sendo promissora na fitorremediação de sítios com resíduos do imazapic.
\end{abstract}

PALAVRAS-CHAVE: imidazolinonas, metabolismo, persistência, fitointoxicação.

\begin{abstract}
Imazapic can cause visual intoxication in sensitive crops sown in rotation given its long residual effect in the soil and represents ecotoxicological risks in aquatic environments. In this scenario, phytoremediation is an innovative proposal as an alternative method for removing organic pollutants, with its success depending on the careful selection of plants with favorable characteristics. The objective of this study was to identify imazapic-tolerant plant species for potential use in phytoremediation programs. Eight experiments were conducted in a greenhouse using the species Panicum maximum, Crotalaria juncea, Stylosanthes spp., Cajanus cajan, Dolichos lablab, Pennisetum glaucum, Mucuna aterrima, and Raphanus sativus, considering five doses of imazapic $\left(0 ; 58.33 ; 87.5 ; 175\right.$ and $\left.350 \mathrm{~g} \mathrm{ha}^{-1}\right)$. The treatments were delineated in randomized blocks with four replicates. The symptoms of visual toxicity, height, and dry mass of the plants were determined at 30 and 60 days after plant emergence and at the end of the experiment. The species $M$. aterrima, $C$. cajan, and $D$. lablab presented no visual symptoms of phyto-intoxication. $M$. aterrima promoted greater dry mass production when cultivated as a control, and exposure to imazapic did not affect this characteristic. Height and dry mass reduction required higher doses in tolerant species, although the results were not significant for $D$. lablab and $C$. cajan. Among the studied species, $M$. aterrima stood out for its high biomass production, promising phytoremediation of sites with imazapic residues.
\end{abstract}

KEYWORDS: imidazolinonas, metabolism, persistence, phytointoxication.

\section{INTRODUÇÃO}

As imidazolinonas têm sido amplamente utilizadas em mais de 200 países como herbicidas seletivos 
pré ou pós emergentes no controle de um amplo espectro de plantas daninhas e são caracterizadas por taxas de aplicação relativamente baixas, persistência no solo, meia-vida média de 120 dias e baixa toxicidade em mamíferos ( $\left(\mathrm{LL}_{50}>5 \mathrm{~g} \mathrm{~kg}^{-1}\right.$ ) (DOR et al. 2016). O mecanismo de ação das imidazolinonas consiste na inibição da acetolactato sintase (ALS), a primeira enzima envolvida na biossíntese de aminoácidos de cadeia ramificada, valina, leucina e isoleucina (VEGA et al. 2012, PÉREZ-IGLESIAS et al. 2018).

Pertencente às imidazolinonas, o imazapic tem sido recomendado no Brasil para o controle de plantas daninhas nas culturas de cana-de-açúcar (Saccharum officinarum L.) e amendoim (Arachis hypogaea L.) (MAPA/AGROFIT 2019). O baixo pH, o alto teor de matéria orgânica e a baixa umidade do solo são fatores responsáveis por aumentar a persistência dos herbicidas deste grupo químico. Sua elevada atividade residual associada à sensibilidade de certas culturas são indicadores de intoxicação visual em culturas rotacionais (ALISTER \& KOGAN 2005). Redução de 55\% no rendimento de grãos em plantas de arroz (IRGA 417) aos 371 dias após a aplicação (DAA) da mistura de imazethapyr e imazapic $(0,075+$ $0,025 \mathrm{~kg} \mathrm{ha}^{-1}$ ) foram relatadas assim como lesões significativas aos 705 DAA (MARCHESAN et al. 2010). Também reduções de 35, 47, 49 e 59\% da massa seca da parte aérea, respectivamente, foram observadas para as plantas de milho, pepino, rabanete e tomate semeadas aos 1.100 DAA e tratadas com $100 \mathrm{~g} \mathrm{ha}^{-1} \mathrm{da}$ mistura imazethapyr e imazapic (SOUSA et al. 2012).

Conforme a International Union of Pure and Applied Chemistry (IUPAC), o imazapic possui alta solubilidade em água (2230 $\mathrm{mg} \mathrm{L}^{-1}$ ), baixa constante de dissociação de ácido (pka) de 3,6 e baixo coeficiente de carbono orgânico $\left(\mathrm{K}_{\mathrm{oc}}\right)$ de 137 , características que favorecem sua lixiviação. Na prática, resíduos de imazapic têm sido encontrados em águas subterrâneas e superficiais (SILVA et al. 2011, ZANELLA et al. 2012, DAVIS et al. 2014, MAZLAN et al. 2016, SHAIFUDDIN et al. 2016, ALLAN et al. 2017) e seus efeitos sobre organismos aquáticos são reconhecidos. Impacto seletivo na comunidade de zooplâncton foram observados aos 88 dias de tratamento com a mistura fórmula de imazethapyr e imazapic $\left(75+25 \mathrm{~g} \mathrm{ha}^{-1}\right)$ (REIMCHE et al. 2015). Da mesma forma, efeitos neurotóxico e imunodepressivo foram constatados em populações de peixes Rhamdia quelen expostas por 96 horas à mistura de imazapyr e imazapic $\left(0,488+4,88 \mu \mathrm{g} \mathrm{L}^{-1}\right)$ (GOLOMBIESKI et al. 2016).

$\mathrm{O}$ destino dos pesticidas no ambiente está relacionado à presença de vegetação. Poluentes orgânicos são mais facilmente removidos em solos plantados do que em solos em pousio (COUTINHO \& BARBOSA 2007). Os pesticidas podem ser degradados na rizosfera por enzimas vegetais exsudadas ou por microrganismos associados (RIBEIRO et al. 2015). As plantas podem ainda remover poluentes orgânicos absorvendo-os através das raízes e das folhas (BELO et al. 2011). Uma vez absorvido, esses compostos podem ser bioquimicamente convertidos em metabólitos não fitotóxicos ou inativos (KAWAHIGASHI 2009, SÁNCHEZ et al. 2017, MITTON et al. 2018).

A fitorremediação é uma tecnologia emergente que consiste na utilização de plantas na remoção de contaminantes orgânicos e inorgânicos presentes no solo, água e sedimentos (NISSIM et al. 2018). Nesse sentido, o sucesso da fitorremediação depende da biodisponibilidade dos contaminantes e da seleção criteriosa de plantas com características favoráveis, como alta produção de biomassa, crescimento rápido, adaptação a condições climáticas extremas, fácil estabelecimento, presença de raízes profundas e densas, capacidade de crescer em solos pobres em nutrientes e tolerar concentrações relevantes de contaminantes e múltiplos contaminantes (LUO et al. 2017, GERHARDT et al. 2017). Diante disso, o objetivo deste estudo foi identificar espécies vegetais tolerantes ao imazapic para o uso em programas de remediação.

\section{MATERIAL E MÉTODOS}

Os experimentos foram conduzidos em casa de vegetação climatizada localizada no Instituto Federal Goiano, Campus Rio Verde. As unidades experimentais foram constituídas por vasos de polietileno com capacidade para $6 \mathrm{dm}^{3}$ preenchidos com amostra de solo coletada da área do Campus (17\%48'55" $\mathrm{S} e$ 5056’28” O, altitude de $754 \mathrm{~m}$ ), sem histórico de contaminação. O solo seco ao ar foi submetido ao processo de peneiramento e amostras foram coletadas para serem analisadas física e quimicamente. As principais características do solo foram: $63 \%$ de areia, $5 \%$ de silte, $32 \%$ de argila, textura franco argiloarenosa (média), 15,4 $\mathrm{g} \mathrm{dm}^{-3}$ de $\mathrm{CO}, \mathrm{V}$ de $32 \%$, CTC de $7,1 \mathrm{cmol}_{\mathrm{c}} \mathrm{dm}^{-3}$ e $\mathrm{pH}\left(\mathrm{CaCl}_{2}\right)$ de 5,3 .

A identificação das espécies tolerantes ao imazapic baseou-se no método proposto por MADALÃO et al. (2013). As espécies foram selecionadas com base na tolerância já relatada aos herbicidas do grupo das imidazolinonas (SOUTO et al. 2015) bem como por outras características de interesse agronômico.

Foram conduzidos oito experimentos com as espécies Panicum maximum cv. Tanzânia (capimcolonião), Crotalaria juncea cv. Comum (crotalária), Stylosanthes spp. cv. Campo Grande (estilosantes), 
Cajanus cajan cv. Fava Larga (feijão-guandu), Dolichos lablab cv. Rongai (lablab), Pennisetum glaucum cv. BRS 1501 (milheto), Mucuna aterrima cv. Mucuna Preta (mucuna-preta) e Raphanus sativus cv. Comum (nabo forrageiro). Sementes foram adquiridas da Brseeds Produção e Comércio de Sementes Ltda.

Os tratamentos constaram de cinco doses de imazapic $\left(0 ; 58,33 ; 87,5 ; 175\right.$ e $\left.350 \mathrm{~g} \mathrm{ha}^{-1}\right)$ correspondentes a $0,1 / 3,1 / 2,1$ e 2 vezes a dose comercial, respectivamente, segundo MAPA/AGROFIT (2019) delineados em blocos ao acaso com quatro repetições. Ao término da semeadura das espécies, o imazapic foi aplicado em pré-emergência, utilizando um pulverizador de precisão, pressurizado com gás carbônico $\left(\mathrm{CO}_{2}\right)$. O volume de aplicação foi de $250 \mathrm{~L} \mathrm{ha}^{-1}$. Após 10 dias de emergência das plântulas, foi realizado o desbaste deixando-se três plantas por vaso. Procedeu-se irrigação diária para a manutenção do suprimento hídrico das plantas e aplicações a cada 15 dias na dose de $20 \mathrm{~g} \mathrm{~L}^{-1}$ de fertilizante comercial Forth Jardim ${ }^{\circledR}$ da marca Tecnutri do Brasil, contendo N: 13; $\mathrm{P}_{2} \mathrm{O}_{5}: 5 ; \mathrm{K}_{2} \mathrm{O}: 13 ; \mathrm{B}: 0,04 ; \mathrm{Ca}: 1 ; \mathrm{Cu}: 0,05 ; \mathrm{S}: 5$; Fe: 0,2; Mg: 1; Mn: 0,08; Mo: 0,005 e Zn: 0,15\%, aplicando $50 \mathrm{~mL}$ em cada vaso.

Aos 30 e 60 dias após a emergência (DAE) foram avaliados os sintomas de intoxicação visual (\%) e a altura de plantas $(\mathrm{cm})$. A intoxicação nas plantas foi determinada por avaliação visual, utilizando escala percentual entre 0 (sem lesão) a 100\% (morte completa da planta), conforme o proposto por SBCPD (1995). A medição da altura foi analisada por escala graduada, tendo como referência o meristema apical para as dicotiledôneas e a última folha expandida para as monocotiledôneas.

Aos $60 \mathrm{DAE}$, as espécies foram retiradas das unidades experimentais, procedendo-se a separação da parte aérea e do sistema radicular, sendo o material vegetal encaminhado para estufa de secagem com circulação de ar forçado a $70 \stackrel{\circ}{\circ}$ por $72 \mathrm{~h}$, seguido de pesagem para a determinação da massa seca.

As médias referentes à altura de plantas e as massas secas da parte aérea (MSPA) e de raiz (MSR) obtidas pela testemunha de cada espécie (dose zero), dentro de cada dose foram transformadas, atribuindo-se índice igual a 1.

Os resultados foram submetidos à análise de variância, ao nível de $5 \%$ de significância e à análise de regressão. A escolha dos modelos baseou-se na significância dos parâmetros, resposta biológica e nos valores de $\mathrm{R}^{2}$. Posteriormente, os modelos definidos foram submetidos à análise de identidade de modelos e igualdade de parâmetros.

\section{RESULTADOS}

Os resultados das variáveis fitointoxicação avaliadas aos 30 e aos 60 DAE, dos índices de altura e de massa seca da parte aérea e de raiz das espécies Panicum maximum, Crotalaria juncea, Stylosanthes spp. e Cajanus cajan em função de doses do herbicida imazapic são apresentados na Tabela 1. Aos 30 e 60 DAE, os resultados obtidos para todos os parâmetros avaliados em $P$. maximum se ajustaram ao modelo logístico. Na primeira avaliação realizada aos $30 \mathrm{DAE}$, a dose de $86,13 \mathrm{~g} \mathrm{ha}^{-1}$ foi suficiente para promover $50 \%$ dos sintomas de fitointoxicação e a partir da dose de $131,25 \mathrm{~g} \mathrm{ha}^{-1}$ os efeitos se estabilizaram com valores próximos a $90 \%$. Aos $60 \mathrm{DAE}$, os sintomas de fitointoxicação foram atenuados, sendo $50 \%$ dos efeitos observados na dose de $158,59 \mathrm{~g} \mathrm{ha}^{-1}$.

Os altos níveis de intoxicação apresentados aos 30 DAE nas plantas de $P$. maximum refletiram na altura nessa mesma época, constatando-se $50 \%$ de reduções no porte das plantas na dose de $112,11 \mathrm{~g}$ $\mathrm{ha}^{-1}$. Aos $60 \mathrm{DAE}$, foi observado plantas de maior altura, em que a dose de $172,27 \mathrm{~g} \mathrm{ha}^{-1}$ representou $50 \%$ da resposta. Quanto a produção de massa seca, o incremento das doses de imazapic promoveu reduções expressivas nesse parâmetro, sendo as doses de 88,87 e $82,03 \mathrm{~g} \mathrm{ha}^{-1}$ suficientes para inibir em $50 \%$ a produção de MSPA e MSR, respectivamente, com reduções acima de $80 \%$ em doses superiores a $175 \mathrm{~g}$ $\mathrm{ha}^{-1}$.

Modelos lineares se ajustaram aos resultados de fitointoxicação, índices de altura e massa seca (Tabela 1) para Crotalaria juncea, em ambas as épocas avaliadas. Aos $30 \mathrm{DAE}$, cada $\mathrm{g} \mathrm{ha}^{-1}$ do herbicida promoveu incremento nos sintomas de fitointoxicação de $0,008 \%$. Comparado à testemunha, o aumento dos sintomas representou $25,43 \%$ na maior dose de $350 \mathrm{~g} \mathrm{ha}^{-1}$. Aos $60 \mathrm{DAE}$, as plantas de C. juncea apresentaram recuperação das lesões apresentando incremento médio sobre a fitointoxicação de 0,005\% para cada $\mathrm{g} \mathrm{ha}^{-1}$ de herbicida aplicado.

Em relação à altura de $C$. juncea avaliada aos 30 DAE, houve decréscimos lineares de $0,09 \%$ para cada $\mathrm{g} \mathrm{ha}^{-1}$. Reduções variando de 14,73 e 30,60\% foram verificadas na altura das plantas a partir da dose de $175 \mathrm{~g} \mathrm{ha}^{-1}$. Aos $60 \mathrm{DAE}$, as plantas apresentaram menor porte em relação à testemunha, observando-se decréscimos lineares de $0,012 \%$ para cada $\mathrm{g} \mathrm{ha}^{-1}$ do herbicida aplicado. Ao avaliar a produção de massa seca, o aumento da dose de imazapic promoveu reduções unitárias ( $\mathrm{g} \mathrm{ha}^{-1}$ de herbicida aplicado) de $0,14 \mathrm{e}$ 0,18\% para os índices MSPA e MSR, respectivamente. 
Tabela 1. Intoxicação em plantas aos 30 dias (FT_30) e aos 60 após a emergência (FT_60), índice de altura avaliados aos 30 dias (IA_30) e aos 60 após a emergência (IA_60), índice de massa seca da parte aérea (MSPA) e de raiz (MSR) de Panicum maximum, Crotalaria juncea, Stylosanthes spp., e Cajanus cajan em função de doses do herbicida imazapic.

Table 1. Intoxication in plants at 30 days (FT_30) and 60 days after emergence (FT_60), height index evaluated at 30 days (IA_30) and 60 days after emergence (IA_60), shoot (MSPA) and root dry mass index (MSR) of Panicum maximum, Crotalaria juncea, Stylosanthes spp., and Cajanus cajan as a function of doses of the imazapic herbicide.

\begin{tabular}{|c|c|c|c|c|c|c|c|}
\hline \multirow{2}{*}{ Variáveis } & \multicolumn{5}{|c|}{ Doses $\left(\mathrm{g} \mathrm{ha}^{-1}\right)$} & \multirow{2}{*}{ Equação } & \multirow{2}{*}{$\mathrm{R}^{2}$} \\
\hline & 0 & 58,33 & 87,5 & 175 & 350 & & \\
\hline & \multicolumn{7}{|c|}{ Panicum maximum } \\
\hline FT_30 & 0 & 2,92 & 54,17 & 89,25 & 89,17 & $\hat{Y}=89,21 /\left(1+\exp ^{(-(x-84,18) / 7,63)}\right)$ & $1,00^{*}$ \\
\hline FT_60 & 0 & 6,67 & 26,67 & 54,17 & 78,33 & $\hat{Y}=78,18 /\left(1+\exp ^{(-(x-133,81) / 44,05)}\right)$ & $0,98^{*}$ \\
\hline IA_30 & 1 & 0,95 & 0,57 & 0,20 & 0,11 & $\hat{Y}=1,13 /\left(1+\exp ^{(-(x-103,79) /-42,70)}\right)$ & $0,95^{*}$ \\
\hline IA_60 & 1 & 1,14 & 0,90 & 0,46 & 0,16 & $\hat{Y}=1,10 /\left(1+\exp ^{(-(x-164,13) /-42,79)}\right)$ & $0,96^{*}$ \\
\hline MSPA & 1 & 0,92 & 0,54 & 0,10 & 0,08 & $\hat{Y}=1,00 /\left(1+\exp ^{(-(-x-89,45) /-13,17)}\right)$ & $0,98^{*}$ \\
\hline \multirow[t]{2}{*}{ MSR } & 1 & 0,60 & 0,47 & 0,09 & 0,07 & $\hat{Y}=1,22 /\left(1+\exp ^{(-(x-66,13) /-44,23)}\right)$ & $0,99^{*}$ \\
\hline & \multicolumn{7}{|c|}{ Crotalaria juncea } \\
\hline FT_30 & 0 & 2,50 & 0,00 & 15,00 & 25,00 & $\hat{Y}=-2,024-0,0784 x$ & $0,92^{*}$ \\
\hline FT_60 & 0 & 1,25 & 0,00 & 6,25 & 15,00 & $\hat{Y}=-1,6456-0,0454 x$ & $0,95^{*}$ \\
\hline IA_30 & 1 & 0,99 & 0,94 & 0,80 & 0,71 & $\hat{Y}=1,0113-0,0009 x$ & $0,93^{*}$ \\
\hline IA_60 & 1 & 1,02 & 0,94 & 0,72 & 0,64 & $\hat{Y}=1,0227-0,0012 x$ & $0,87^{*}$ \\
\hline MSPA & 1 & 0,91 & 0,82 & 0,70 & 0,49 & $\hat{Y}=0,9801-0,0014 x$ & $0,98^{*}$ \\
\hline \multirow[t]{2}{*}{ MSR } & 1 & 0,77 & 0,87 & 0,66 & 0,33 & $\hat{Y}=0,9716-0,0018 x$ & $0,95^{*}$ \\
\hline & \multicolumn{7}{|c|}{ Stylosanthes spp. } \\
\hline FT_30 & 0 & 11,00 & 9,17 & 46,25 & 62,75 & $\hat{Y}=0,2047-0,1910 x$ & $0,92^{*}$ \\
\hline FT_60 & 0 & 12,92 & 2,50 & 32,08 & 51,67 & $\hat{Y}=-0,7223-0,1532 x$ & $0,91^{*}$ \\
\hline IA_30 & 1 & 1,16 & 1,09 & 0,76 & 0,64 & $\hat{Y}=1,1197-0,0014 x$ & $0,74^{*}$ \\
\hline IA_60 & 1 & 1,13 & 1,15 & 0,62 & 0,49 & $\hat{Y}=1,1389-0,0019 x$ & $0,74^{*}$ \\
\hline MSPA & 1 & 1,01 & 0,96 & 0,35 & 0,34 & $\hat{Y}=1,02 /\left(1+\exp ^{(-(x-158,74) /-26,10)}\right)$ & $0,77^{*}$ \\
\hline \multirow[t]{2}{*}{ MSR } & 1 & 0,80 & 0,83 & 0,61 & 0,45 & $\hat{Y}=0,9386-0,0015 x$ & $0,93^{*}$ \\
\hline & \multicolumn{7}{|c|}{ Cajanus cajan } \\
\hline FT_30 & 0 & 1,67 & 2,50 & 6,67 & 3,75 & $\bar{Y}=2,92$ & - \\
\hline FT_60 & 0 & 0,00 & 1,67 & 0,42 & 0,00 & $\bar{Y}=0,42$ & - \\
\hline IA_30 & 1 & 1,12 & 1,11 & 1,08 & 1,14 & $\bar{Y}=1,12$ & - \\
\hline IA_60 & 1 & 1,18 & 1,21 & 1,19 & 1,22 & $\bar{Y}=1,18$ & - \\
\hline MSPA & 1 & 1,08 & 1,23 & 1,02 & 1,11 & $\bar{Y}=1,08$ & - \\
\hline MSR & 1 & 1,16 & 1,23 & 0,97 & 1,28 & $\bar{Y}=1,12$ & - \\
\hline
\end{tabular}

${ }^{*}$ significativo a $5 \%$ pelo teste $\mathrm{F}$.

Em plantas de Stylosanthes, os sintomas de fitointoxicação e índice de altura foram ajustados aos modelos lineares para as avaliações realizadas em ambas as épocas (Tabela 1). Aos 30 DAE, para cada $g$ $\mathrm{ha}^{-1}$ do herbicida aplicado, a intoxicação aumentou em $0,19 \%$. Comparado à testemunha, os aumentos dos sintomas representaram 33,63 e 67,06\%, respectivamente, nas doses de 175 e $350 \mathrm{~g} \mathrm{ha}^{-1}$. Aos $60 \mathrm{DAE}$, as plantas de Stylosanthes apresentaram ligeira recuperação dos sintomas visuais de intoxicação. Todavia, mantendo tendência observada na avaliação inicial, os sintomas foram superiores a $30 \%$ nas doses acima de $175 \mathrm{~g} \mathrm{ha}^{-1}$ (Tabela 1 ).

$O$ incremento das doses promoveu redução linear na altura das plantas de Stylosanthes em ambas as épocas (Tabela 1). Aos $30 \mathrm{DAE}$, a altura apresentou perdas unitárias de $0,14 \%$, o que caracterizou redução de $36,65 \%$ na maior dose de $350 \mathrm{~g} \mathrm{ha}^{-1}$ em relação à testemunha. Aos $60 \mathrm{DAE}$, para as variáveis índice de altura e MSR, o incremento da dose promoveu decréscimos lineares de 0,19 e $0,15 \%$, respectivamente, para cada $\mathrm{g} \mathrm{ha}^{-1}$ do herbicida aplicado. Enquanto os efeitos na MSPA foram mais representativos a partir da dose de $133,98 \mathrm{~g} \mathrm{ha}^{-1}$ e sua resposta ajustada ao modelo logístico, com redução de $50 \%$ de MSPA na dose de 159,96 $\mathrm{g} \mathrm{ha}^{-1}$ e ausência na produção desta, nas doses acima de $278,91 \mathrm{~g}$ 
$\mathrm{ha}^{-1}$ (Tabela 1).

Em plantas de C. cajan, os níveis de fitointoxicação foram insignificantes em ambas as épocas, permanecendo entre 0,42 e 2,92\% (Tabela 1). Essa tendência refletiu na resposta das variáveis altura que apresentaram valores estatisticamente semelhantes à testemunha, aos 30 e aos 60 DAE, bem como de massa seca da parte aérea e de raiz.

Em plantas de $D$. lablab, o incremento das doses não promoveu sintomas de intoxicação em ambas as épocas avaliadas (Tabela 2). Para esse parâmetro, os valores permaneceram entre 2,91 e 4,03\%, o que influenciou a altura e os índices MSPA e MSR, que não foram afetados com as doses do herbicida. Todavia, em plantas de $P$. glaucum, o modelo logístico explicou o efeito das doses sobre os sintomas de intoxicação em ambas as épocas avaliadas (Tabela 2). Aos $30 \mathrm{DAE}$, a dose de 125,78 $\mathrm{g} \mathrm{ha}^{-1}$ promoveu $50 \%$ dos sintomas visuais. Aos $60 \mathrm{DAE}, 50 \%$ da resposta foi obtida na dose de $151,76 \mathrm{~g} \mathrm{ha}^{-1}$ e a partir da dose de $250,02 \mathrm{~g} \mathrm{ha}^{-1}$ as plantas de $P$. glaucum apresentaram tendência de estabilização dos efeitos, com valores de intoxicação próximos a 75\% (Tabela 2).

Tabela 2. Intoxicação em plantas aos 30 dias (FT_30) e aos 60 após a emergência (FT_60), índice de altura avaliados aos 30 dias (IA_30) e aos 60 após a emergência (IA_60), índice de massa seca da parte aérea (MSPA) e de raiz (MSR) de Dolichos lablab, Pennisetum glaucum, Mucuna aterrima e Raphanus sativus em função de doses do herbicida imazapic.

Table 2. Intoxication in plants at 30 days (FT_30) and 60 days after emergence (FT_60), height index evaluated at 30 days (IA_30) and 60 days after emergence (IA_60), shoot (MSPA) and root dry mass index (MSR) of Dolichos lablab, Pennisetum glaucum, Mucuna aterrima and Raphanus sativus as a function of doses of the imazapic herbicide.

\begin{tabular}{|c|c|c|c|c|c|c|c|}
\hline \multirow{2}{*}{ Variáveis } & \multicolumn{5}{|c|}{ Doses $\left(\mathrm{g} \mathrm{ha}^{-1}\right)$} & \multirow{2}{*}{ Equação } & \multirow{2}{*}{$\mathrm{R}^{2}$} \\
\hline & 0 & 58,33 & 87,5 & 175 & 350 & & \\
\hline & \multicolumn{7}{|c|}{ Dolichos lablab } \\
\hline FT_30 & 0 & 3,75 & 2,50 & 4,17 & 4,17 & $\bar{Y}=2,91$ & - \\
\hline FT_60 & 0 & 3,75 & 6,67 & 6,67 & 3,33 & $\bar{Y}=4,03$ & - \\
\hline IA_30 & 1 & 1,02 & 0,90 & 0,84 & 0,94 & $\bar{Y}=0,94$ & - \\
\hline IA_60 & 1 & 1,02 & 0,89 & 0,89 & 0,97 & $\bar{Y}=0,99$ & - \\
\hline MSPA & 1 & 1,02 & 0,98 & 0,98 & 1,00 & $\bar{Y}=0,99$ & - \\
\hline \multirow[t]{2}{*}{ MSR } & 1 & 0,95 & 0,75 & 0,64 & 0,90 & $\bar{Y}=0,84$ & - \\
\hline & \multicolumn{7}{|c|}{ Pennisetum glaucum } \\
\hline FT_30 & 0 & 3,75 & 13,33 & 68,75 & 68,17 & $\hat{Y}=68,99 /\left(1+\exp ^{(-(x-109,69) / 15,68)}\right)$ & $0,99^{*}$ \\
\hline $\mathrm{FT}-60$ & 0 & 19,17 & 5,67 & 63,33 & 74,17 & $\hat{Y}=75,77 /\left(1+\exp ^{(-(x-131,36) / 26,67)}\right)$ & $0,96^{*}$ \\
\hline IA_30 & 1 & 0,97 & 0,89 & 0,50 & 0,37 & $\hat{Y}=1,0158-0,020 x$ & $0,88^{*}$ \\
\hline IA_60 & 1 & 0,91 & 1,01 & 0,36 & 0,21 & $\hat{Y}=1,0434-0,026 x$ & $0,84^{*}$ \\
\hline MSPA & 1 & 0,65 & 0,71 & 0,20 & 0,09 & $\hat{Y}=1,32 /\left(1+\exp ^{(-(x-75,97) /-70,23)}\right)$ & $0,95^{*}$ \\
\hline \multirow[t]{2}{*}{ MSR } & 1 & 0,66 & 0,44 & 0,10 & 0,15 & $\hat{Y}=1,32 /\left(1+\exp ^{(-(x-55,88) /-48,65)}\right)$ & $0,96^{*}$ \\
\hline & \multicolumn{7}{|c|}{ Mucuna aterrima } \\
\hline FT_30 & 0 & 3,75 & 2,08 & 12,08 & 8,58 & $\bar{Y}=8,70$ & - \\
\hline FT_60 & 0 & 5,00 & 12,50 & 8,33 & 17,92 & $\bar{Y}=5,30$ & - \\
\hline IA_30 & 1 & 0,97 & 0,77 & 0,61 & 0,47 & $\hat{Y}=0,9573-0,0018 x$ & $0,89^{*}$ \\
\hline IA_60 & 1 & 0,99 & 0,79 & 0,64 & 0,54 & $\hat{Y}=0,9797-0,0014 x$ & $0,85^{*}$ \\
\hline MSPA & 1 & 1,02 & 0,91 & 0,96 & 0,77 & $\hat{Y}=1,02013-0,0007 x$ & $0,82^{*}$ \\
\hline \multirow[t]{2}{*}{ MSR } & 1 & 1,05 & 0,93 & 0,92 & 0,74 & $\hat{Y}=1,0388-0,0008 x$ & $0,87^{*}$ \\
\hline & \multicolumn{7}{|c|}{ Raphanus sativus } \\
\hline FT_30 & 0 & 52,92 & 95,42 & 96,92 & 99,42 & $\hat{Y}=98,16 /\left(1+\exp ^{(-(x-56,99) / 8,59)}\right)$ & $0,99^{*}$ \\
\hline FT_60 & 0 & 63,33 & 96,71 & 97,17 & 100,00 & $\hat{Y}=98,56 /\left(1+\exp ^{(-(x-53,29) / 8,60)}\right)$ & $0,99^{*}$ \\
\hline IA_30 & 1 & 0,40 & 0,15 & 0,16 & 0,07 & $\hat{Y}=0,997 \exp ^{-0,017 x}$ & $0,96^{*}$ \\
\hline IA_60 & 1 & 0,13 & 0,02 & 0,01 & 0,00 & $\hat{Y}=1,000 \exp ^{-0,038 x}$ & $0,99^{*}$ \\
\hline MSPA & 1 & 0,25 & 0,01 & 0,01 & 0,00 & $\hat{Y}=1,003 \exp ^{-0,028 x}$ & $0,98^{*}$ \\
\hline MSR & 1 & 0,19 & 0,01 & 0,00 & 0,00 & $\hat{Y}=1,002 \exp ^{-0,032 x}$ & $0,99^{*}$ \\
\hline
\end{tabular}

${ }^{*}$ significativo a $5 \%$ pelo teste $\mathrm{F}$. 
Reduções lineares na altura das plantas de $P$. glaucum foram observadas em ambas as épocas (Tabela 2). Aos $30 \mathrm{DAE}$, para cada $\mathrm{g}$ ha $^{-1}$ de herbicida aplicado observou-se redução de $0,20 \%$ no porte das plantas. Comparado à testemunha, o decréscimo na altura representou $68,96 \%$ na dose de $350 \mathrm{~g} \mathrm{ha}^{-1}$. Aos 60 DAE, o aumento das doses de imazapic ocasionou maior incremento na redução da altura em relação a avaliação inicial. Cada $\mathrm{g}$ ha $^{-1}$ do herbicida aplicado promoveu decréscimo de $0,26 \%$ e caracterizou reduções de 40,47 e $85,28 \%$ nas doses de 175 e $350 \mathrm{~g} \mathrm{ha}^{-1}$.

O modelo logístico descreveu satisfatoriamente a relação entre as doses de imazapic e os índices de massa seca nas plantas de $P$. glaucum aos 60 DAE (Tabela 2). As doses de 110,74 e 79,29 $\mathrm{g} \mathrm{ha}^{-1}$ ocasionaram $50 \%$ de redução da MSPA e MSR, respectivamente. Para MSR, os valores foram superiores a $90 \%$ a partir da dose de $175 \mathrm{~g} \mathrm{ha}^{-1}$.

Aos 30 e $60 \mathrm{DAE}$, os sintomas visuais de intoxicação observados nas plantas de $M$. aterrima foram insignificantes, permanecendo entre 5,3 e 8,7\% (Tabela 2). Todavia, o incremento das doses promoveu reduções lineares sobre as demais variáveis estudadas e em ambas as épocas. Aos $30 \mathrm{DAE}$, a altura das plantas apresentou decréscimo de $0,16 \%$ para cada $\mathrm{g} \mathrm{ha}^{-1}$ de herbicida, com reduções variando entre $30 \mathrm{e}$ $58 \%$ nas doses acima de $175 \mathrm{~g} \mathrm{ha}^{-1}$. Aos $60 \mathrm{DAE}$, os efeitos foram ligeiramente atenuados e a redução na altura foi de $0,14 \%$ para cada $\mathrm{g} \mathrm{ha}^{-1}$ aplicado. A produção de MSPA e MSR apresentou perdas unitárias de 0,07 e $0,08 \%$, respectivamente, e caracterizou reduções inferiores a $25 \%$ nas plantas tratadas com a maior dose (350 $\mathrm{g} \mathrm{ha}^{-1}$ ) de imazapic (Tabela 2).

As plantas de $R$. sativus mostraram-se altamente suscetíveis ao aumento das doses de imazapic, independentemente da época avaliada (Tabela 2). Os resultados de intoxicação visual foram ajustados pelo modelo logístico, sendo 50\% dos sintomas visuais ocasionados em doses de 50,32 e 53,32 $\mathrm{g} \mathrm{ha}^{-1}$ aos 30 e 60 DAE, respectivamente. A partir da dose de 95,70 $\mathrm{g} \mathrm{ha}^{-1}$ as plantas apresentaram tendência de estabilização dos efeitos próximos de 100\% (Tabela 2).

Modelos exponenciais decrescentes se ajustaram aos índices de altura, MSPA e MSR em ambas as épocas (Tabela 2). Aos 30 DAE, a altura das plantas apresentou reduções superiores a $80 \%$ em doses acima de $94,33 \mathrm{~g} \mathrm{ha}^{-1}$. Aos 60 DAE, os efeitos foram mais acentuados e $100 \%$ de redução nesses parâmetros (altura, MSPA e MSR) foi verificado em doses abaixo de $175 \mathrm{~g} \mathrm{ha}^{-1}$.

Entre as espécies avaliadas, a maior produção de massa seca foi determinada em plantas de $M$. aterrima quando cultivada como testemunha (Tabela 3). Além dessa característica, $M$. aterrima não apresentou sintomas visuais de fitointoxicação e manteve alta produção de massa seca da parte aérea e raiz, critérios utilizados neste estudo para a identificação das espécies tolerantes ao imazapic.

Tabela 3. Massa seca das espécies bioindicadoras cultivadas como testemunhas.

Table 3. Dry mass of bioindicator species grown as controls.

\begin{tabular}{lc}
\hline Espécies bioindicadoras & Massa seca (g por planta) \\
\hline Panicum maximum cv. Tanzânia (Capim-colonião) & 13,78 \\
Crotalaria juncea cv. Comum (Crotalaria) & 12,74 \\
Stylosanthes spp. cv. Campo Grande (Estilosantes) & 1,80 \\
Cajanus cajan cv. Fava Larga (Feijão guandu) & 9,65 \\
Dolichos lablab cv. Rongai (Lablab) & 9,88 \\
Pennisetum glaucum cv. BRS 1501 (Milheto) & 16,64 \\
Mucuna aterrima cv. Mucuna Preta (Mucuna-preta) & 23,55 \\
Raphanus sativus cv. Comum (Nabo forrageiro) & 16,20 \\
\hline
\end{tabular}

\section{DISCUSSÃO}

As espécies $M$. aterrima, $C$. cajan e $D$. lablab não apresentaram sintomas visuais de intoxicação com 0 incremento das doses de imazapic, independentemente da época avaliada. Alguns trabalhos relatam o potencial de $C$. cajan quanto à tolerância aos herbicidas diclosulam, tebuthiuron e sulfentrazone (PIRES et al. 2008, MADALÃO et al. 2012, MONQUERO et al. 2013). D. lablab foi uma das espécies selecionadas por MADALÃO et al. (2013) como tolerante ao sulfentrazone em doses até $400 \mathrm{~g} \mathrm{ha}^{-1}$.

Entre as espécies tolerantes, $M$. aterrima foi a que apresentou maior produção de massa seca quando cultivada como testemunha, sendo esse parâmetro 2,4 vezes superior ao verificado em $C$. cajan e $D$. lablab. A exposição ao imazapic pouco afetou essa característica em $M$. aterrima que apresentou reduções inferiores a $25 \%$ na biomassa na maior dose do herbicida, contrariamente às espécies suscetíveis. 
Em campo experimental, M. aterrima apresentou potencial em fitorremediar fomesafen e sulfentrazone (ALVES et al. 2018). A capacidade de fitoextração de hidrocarbonetos associada ao maior acúmulo de biomassa também foi reportada para essa espécie (NWAICHI et al. 2009, NWAICHI \& AYALOGU 2010). A alta produção de biomassa e tolerância ao composto alvo a ser removido são requisitos necessários para a eficiência da fitorremediação (MARCACCl et al. 2006).

Nesse estudo, a tolerância pode ser atribuída ao metabolismo diferencial apresentado pelas espécies ao imazapic. Um estudo sobre o metabolismo do imazapic em seis cultivares de soja mostrou que a cultivar tolerante apresentou maior absorção e rápida translocação do herbicida. No entanto, em função das diferenças que ocorreram entre 48 e $96 \mathrm{~h}$ da aplicação foi atribuído ao metabolismo a resposta diferencial (NEWSOM et al. 1995). Em cultivar tolerante de trigo foi relatado que $67,74 \%$ de imazamox foi rapidamente metabolizado na maior dose do herbicida $\left(200 \mathrm{~g} \mathrm{ha}^{-1}\right)$ às 120 horas após a aplicação, enquanto na cultivar sensível apenas $0,64 \%$ foi metabolizado nesse mesmo período (ROJANO-DELGADO et al. 2015). O metabolismo reduz a quantidade de herbicida intracelular que pode inibir a enzima ALS promovendo maior tolerância às plantas tratadas com imidazolinonas (ROJANO-DELGADO et al. 2012).

O mecanismo de tolerância é baseado na capacidade da planta em rapidamente metabolizar o herbicida, convertendo-o a um produto não fitotóxico (YU et al. 2004). Uma vez absorvido, o herbicida pode ser ativado por enzimas monooxigenases ou peroxidases do citocromo $\mathrm{P}_{450}$ responsável por mediar reações de oxidação e hidroxilação, aumentando a polaridade da molécula e reduzindo sua toxicidade. Essas reações fornecem sítios de ligação para a conjugação com carboidratos (SIMINSZKY 2006, KASPAR et al. 2011, SCHWITZGUÉBEL 2017). No entanto, poucos genes associados ao metabolismo desses herbicidas foram identificados (ZHAO et al. 2017).

Nas espécies suscetíveis ao imazapic, os sintomas visuais de fitointoxicação incluíam deformações na parte aérea, clorose e necrose foliar. Clorose em folhas de girassol tratadas com imazamox foi relacionado ao seu conteúdo de clorofila significativamente reduzido (BALABANOVA et al. 2016). Nas espécies suscetíveis, $P$. maximum, $C$. juncea e Stylosanthes, as lesões aumentaram com as doses de imazapic e reduziram com o tempo após a aplicação. GARCíA-GARIJO et al. (2013) relataram 50\% de inibição da atividade de ALS em tecidos jovens aos sete dias após o tratamento com imazamox, enquanto nas folhas maduras esse efeito não foi observado. Em cada órgão da planta, os aminoácidos de cadeia ramificada são sintetizados principalmente nos tecidos jovens. Assim, os primeiros efeitos promovidos pelos herbicidas inibidores da ALS ocorrem nos tecidos meristemáticos. Tecidos maduros possuem maiores quantidades de aminoácidos e reservas de proteína que podem ser catabolizadas, resultando em maior tempo de exposição para a ocorrência do efeito tóxico (ZHOU et al. 2007).

Conforme os resultados, a exposição ao imazapic influenciou de forma distinta o crescimento e a biomassa das plantas. Em plantas de P. maximum, Stylosanthes, P. glaucum, R. sativus e C. juncea, a redução da altura e massa seca foi alta nas menores doses de imazapic. Nessas espécies, a massa seca foi mais sensível às doses do herbicida do que a altura das plantas. Resultados semelhantes foram relatados por SU et al. (2016) com mudas de milho expostas à concentrações de até $800 \mathrm{\mu g} \mathrm{kg}^{-1} \mathrm{de}$ imazapic. Enquanto nas demais espécies, a redução desses parâmetros exigiu doses maiores, embora os resultados não tenham sido estatisticamente significativos para $D$. lablab e $C$. cajan.

A resposta diferencial das espécies ao imazapic pode ser explicada por diferentes mecanismos físicos, moleculares e fisiológicos envolvidos na tolerância ao herbicida (TAN et al. 2005). A recuperação da clorofila favorecida pelo metabolismo, a restituição de proteínas dos cloroplastos, a exclusão do herbicida, além da possível substituição de aminoácido no sítio de ação, são alguns dos mecanismos envolvidos na tolerância às imidazolinonas (JIMÉNEZ et al. 2015). Efeitos negativos apresentados por algumas espécies de plantas à diferentes níveis de imidazolinonas têm sido relatados. MATOCHA et al. (2003) verificaram redução na altura de plantas de algodão nas doses entre 140 e $210 \mathrm{~g} \mathrm{ha}^{-1}$ de imazapic no ano seguinte da aplicação. YORK et al. (2000) observaram 58\% de lesões, atraso na maturação e $44 \%$ de redução do rendimento do algodão na dose de $140 \mathrm{~g} \mathrm{ha}^{-1}$ de imazapic quando aplicado em pré emergência. A dose de $140 \mathrm{~g} \mathrm{ha}^{-1}$ da mistura formulada com imazapic e imazapyr também reduziu em $72 \%$ a massa seca da parte aérea em plantas de azevém semeadas em sucessão ao arroz Clearfield ${ }^{\circledR}$ (SANTOS et al. 2014). ALISTER \& KOGAN (2005) verificaram efeito residual do imazapic na dose de $119,7 \mathrm{~g} \mathrm{ha}^{-1}$ sobre o crescimento e rendimento de grãos em plantas de azevém e cevada aos 300 dias após a aplicação.

É reconhecida que a atividade microbiana é a principal via de degradação dos herbicidas imidazolinonas (SU et al. 2019). A tolerância ao imazapic também pode ser atribuída à capacidade dessas plantas em associar-se com bactérias fixadoras de nitrogênio e fungos micorrízicos e fitoestimular a microbiota do solo por meio da liberação de exsudatos radiculares (SPOHN et al. 2013, ALVES et al. 2018). 
A tolerância de $L$. multiflorum e $V$. sativa à mistura de imazapyr e imazapic foi atribuída à fitoestimulação da microbiota associada à rizosfera destas espécies (GALON et al. 2014). SOUTO et al. (2015) relataram em média, $94 \%$ de remoção de imazazetapyr e imazapic $\left(4000 \mathrm{~mL} \mathrm{ha}^{-1}\right)$ em solo rizosférico de $S$. aterrimum aos 63 dias de tratamento. LIU et al. (2016) identificaram a cepa A. baumannii IB5, coletada de campo agrícola com histórico de aplicação de imazamox e altamente adaptada a utilizar o herbicida como a única fonte de carbono. Em contraste, amostras de solo coletadas de área com histórico de dois anos de aplicação de imidazolinonas, enriquecidas com misturas formuladas de imazethapyr e imazapic $(75+25 \mathrm{~g}$ $\left.\mathrm{L}^{-1}\right)$ ou imazapyr e imazapic $\left(525+175 \mathrm{~g} \mathrm{~L}^{-1}\right)$ e incubadas por 90 dias, não apresentaram degradação acelerada (BUNDT et al. 2015).

\section{CONCLUSÃO}

Nesse estudo, as espécies Mucuna aterrima, Dolichos lablab e Cajanus cajan apresentaram tolerância ao imazapic, característica requerida na seleção de plantas com potencial fitorremediador. Todavia, embora a exposição ao herbicida promova efeito nas maiores doses sobre a altura e produção de massa seca de Mucuna aterrima, esta espécie é a que mais se destacou pela alta produção de biomassa, sendo esta a mais promissora na fitorremediação do imazapic.

\section{AGRADECIMENTOS}

Ao Instituto Federal Goiano, Campus Rio Verde e a Fundação de Amparo à Pesquisa do Estado de Goiás (FAPEG), pelo apoio financeiro.

\section{REFERÊNCIAS}

ALISTER CA \& KOGAN M. 2005. Efficacy of imidazolinone herbicides applied to imidazolinone resistant maize and their carryover effect on rotational crops. Crop Protection 24: 375-379.

ALLAN HL et al. 2017. Analysis of sugarcane herbicides in marine turtle nesting areas and assessment of risk using in vitro toxicity assays. Chemosphere 185: 656-664.

ALVES C et al. 2018. Selection of species with soil phytoremediation potential after the application of Protox inhibiting herbicides. Planta Daninha 36: e018174765.

BALABANOVA DA et al. 2016. Photosynthetic performance of the imidazolinone resistant sunflower exposed to single and combined treatment by the herbicide imazamox and an amino acid extract. Frontiers in Plant Science 7: 1-10.

BELO AF et al. 2011. Potencial de espécies vegetais na remediação de solo contaminado com sulfentrazone. Planta Daninha 29: 821-828.

BUNDT AC et al. 2015. Imidazolinone degradation in soil in response to application history. Planta Daninha 33: 341 349.

COUTINHO HD \& BARBOSA AR 2007. Fitorremediação: considerações gerais e características de utilização. Silva Lusitana 15: 103-117.

DAVIS AM et al. 2014. The potential benefits of herbicide regulation: a cautionary note for the Great Barrier Reef catchment area. Science Total Environment 490: 81-92.

DOR E et al. 2016. Characterization of the novel tomato mutant HRT, resistant to acetolactate synthase-inhibiting herbicides. Weed Science 64: 348-360.

GALON L et al. 2014. Potential of plant species for bioremediation of soils applied with imidazolinone herbicides. Planta Daninha 32: 719-726.

GARCÍA-GARIJO A et al. 2013. Physiological and biochemical responses of common vetch to the imazamox accumulation. Plant Physiology and Biochemistry 73: 321-325.

GERHARDT KE et al. 2017. Opinion: Taking phytoremediation from proven technology to accepted practice. Plant Science 256: 170-185.

GOLOMBIESKI Jl et al. 2016. Imazapyr + imazapic herbicide determines acute toxicity in silver catfish Rhamdia quelen. Ecotoxicology and Environmental Safety 128: 91-99.

JIMÉNEZ F et al. 2015. Resistance to imazamox in Clearfield soft wheat (Triticum aestivum L.). Crop Protection 78: 1519.

KASPAR M et al. 2011. Selection of a sunflower line with multiple herbicide tolerance that is reversed by the P450 inhibitor malathion. Weed Science 59: 232-237.

KAWAHIGASHI H. 2009. Transgenic plants for phytoremediation of herbicides. Current Opinion in Biotechnology 20: 225-230.

LIU C et al. 2016. Imazamox microbial degradation by common clinical bacteria: Acinetobacter baumannii IB5 isolated from black soil in China shows high potency. Journal of Integrative Agriculture 15: 1798-1807.

LUO $\mathrm{J}$ et al. 2017. Improvement effects of cytokinin on EDTA assisted phytoremediation and the associated environmental risks. Chemosphere 185: 386-393.

MADALÃO JC et al. 2013. Susceptibilidade de espécies de plantas com potencial de fitorremediação do herbicida sulfentrazone. Revista Ceres 60: 111-121. 
MADALÃO JC et al. 2012. Uso de leguminosas na fitorremediação de solo contaminado com sulfentrazone. Pesquisa Agropecuária Tropical 42: 390-396.

MAPA/AGROFIT. 2019. Sistemas de Agrotóxicos Fitossanitários - Consulta Aberta. Disponível em: http://agrofit.agricultura.gov.br/agrofit/. Acesso em: 14 dez. 2019.

MARCACCI S et al. 2006. Conjugation of atrazine in vetiver (Chrysopogon zizanioides Nash) grown in hydroponics. Environmental and Experimental Botany 56: 205-215.

MARCHESAN E et al. 2010. Carryover of imazethapyr and imazapic to nontolerant rice. Weed Technology 24: 6-10.

MATOCHA MA et al. 2003. The persistence of imazapic in peanut (Arachis hypogaea) crop rotations. Weed Technology 17: 325-329.

MAZLAN AZ et al. 2016. Assessment of imazapic presence surface water and groundwater in paddy field area. Jurnal Teknologi 78: 33-37.

MITTON FM et al. 2018. DDTs-induced antioxidant responses in plants and their influence on phytoremediation process. Ecotoxicology and Environmental Safety 147: 151-156.

MONQUERO PA et al. 2013. Seleção de espécies de adubos verdes visando à fitorremediação de diclosulam. Planta Daninha 31: 127-135.

NEWSOM LJ et al. 1995. Absorption, translocation, and metabolismo of AC 263,222 in selected soybean (Glycine max) cultivars. Weed Science 43: 536-540.

NISSIM WG et al. 2018. Phytoremediation of sewage sludge contaminated by trace elements and organic compounds. Environmental Research 164: 356-366.

NWAICHI OE \& AYALOGU OE 2010. Allelopathy as expressed by Mucuna pruriens and the possibility for weed management. International Journal of Plant Physiology and Biochemistry 2: 1-5.

NWAICHI EO et al. 2009. Phytoextracting Cadmium and Copper Using M. pruriens. African Journal Plant Science 3: 277-282.

PÉREZ-IGLESIAS JM et al. 2018. Are the damaging effects induced by the imazethapyr formulation Pivot ${ }^{\circledR} \mathrm{H}$ in $B o a n a$ pulchella (Anura) reversible upon ceasing exposure? Ecotoxicology and Environmental Safety 148: 1-10.

PIRES FR et al. 2008. Avaliação da fitorremediação de tebuthiuron utilizando Crotalaria juncea como planta indicadora. Revista Ciência Agronômica 39: 245-250.

REIMCHE GB et al. 2015. Imazethapyr and imazapic, bispyribac-sodium and penoxsulam: Zooplankton and dissipation in subtropical rice paddy water. Science of the Total Environment 514: 68-76.

RIBEIRO TS et al. 2015. Avaliação do potencial de biorremediação de solos contaminados: método de hidrólise de diacetato de fluoresceína (FDA) como indicador de atividade microbiana. Revista Aquila 13: 105-120.

ROJANO-DELGADO AM et al. 2012. Limited uptake, translocation and enhanced metabolic degradation contribute to glyphosate tolerance in Mucuna pruriens var. utilis plants. Phytochemistry 73: 34-41.

ROJANO-DELGADO AM et al. 2015. Mechanism of imazamox resistance of the Clearfield ${ }^{\circledR}$ wheat cultivar for better weed control. Agronomy for Sustainable Development 35: 639-648.

SÁNCHEZ V et al. 2017. Assessing the phytoremediation potential of crop and grass plants for atrazine-spiked soils. Chemosphere 185: 119-126.

SANTOS LO et al. 2014. Carryover effect of imidazolinone herbicides for crops following rice. American Journal of Plant Sciences 5: 1049-1058.

SCHWITZGUÉBEL JP. 2017. Phytoremediation of soils contaminated by organic compounds: hype, hope and facts. Journal of Soils and Sediments 17: 1492-1502.

SHAIFUDDIN SNM. 2016. Optimization of extraction and detection method for imazapyr and imazapic residues in water, soil and fish tissue samples using high performance liquid chromatography. Jurnal Teknologi 78: 43-48.

SILVA DRO et al. 2011. Ocorrência de agrotóxicos em águas subterrâneas de áreas adjacentes a lavouras de arroz irrigado. Química Nova 34: 748-752.

SIMINSZKY B. 2006. Plant cytochrome P450-mediated herbicide metabolism. Phytochemistry Reviews 5: 445-458.

SBCPD. 1995. Sociedade Brasileira da Ciência das Plantas Daninhas. Procedimentos para instalação, avaliação e análise de experimentos com herbicidas. Londrina: SBCPD. 42p.

SOUSA CP et al. 2012. Growth of residual herbicide (imazethapyr+imazapic) bio-indicators sown in rotation with Clearfield $^{\circledR}$ rice. Planta Daninha 30: 105-111.

SOUTO KM et al. 2015. Phytoremediation of lowland soil contaminated with a formulated mixture of imazethapyr and imazapic. Revista Ciência Agronômica 46: 185-192.

SPOHN M et al. 2013. Microbial gross organic phosphorus mineralization can be stimulated by root exudates - A ${ }^{33} \mathrm{P}$ isotopic dilution study. Soil Biology and Biochemistry 65: 254-263.

SU W et al. 2019. Adsorption and degradation of imazapic in soils under different environmental conditions. PloS one 14: $1-11$.

SU W et al. 2016. Effect of imazapic residues on photosynthetic traits and chlorophyll fluorescence of maize seedlings. Photosynthetica 55: 294-300.

TAN S et al. 2005. Imidazolinone tolerant crops: history, current status and future. Pest Management Science 61: 246257.

VEGA T et al. 2012. Acetohydroxyacid synthase (AHAS) in vivo assay for screening imidazolinone-resistance in sunflower (Helianthus annuus L.). Plant Physiology and Biochemistry 61: 103-107. 
ZANELLA R et al. 2012. Herbicides persistence in rice paddy water in southern Brazil. Herbicides - mechanisms and mode of action. 1.ed. Rijeka: InTech. 23p.

ZHAO B et al. 2017. Non-target site resistance to ALS-inhibiting herbicides in a Sagittaria trifolia L. population. Pesticide Biochemistry and Physiology 140: 79-84.

ZHOU Q et al. 2007. Action mechanisms of acetolactate synthase inhibiting herbicides. Pesticide Biochemistry and Physiology 89: 89-96.

YORK AC et al. 2000. Cotton response to imazapic and imazethapyr applied to a preceding peanut crop. The Journal of Cotton Science 4: 210-216.

YU Q et al. 2004. Tolerance to acetolactate synthase and acetyl-coenzyme A carboxylase inhibiting herbicides in Vulpia bromoides is conferred by two co-existing resistance mechanisms. Pesticide Biochemistry and Physiology 78: 21-30. 